\title{
Pseudo-continuous arterial spin labeling imaging of cerebral blood perfusion asymmetry in drug-naïve patients with first-episode major depression
}

\author{
GUANGDONG CHEN $^{1 *}$, HAIMAN BIAN $^{2 *}$, DEGUO JIANG $^{1 *}$, MINGWEI CUI $^{3}$,

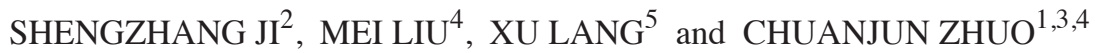

${ }^{1}$ Department of Psychiatry, Wenzhou 7th People's Hospital, Wenzhou, Zhejiang 325000; ${ }^{2}$ Department of Radiology, Tianjin 4th Centre Hospital, Tianjin 300143; ${ }^{3}$ Department of Psychiatry, Tianjin Anning Hospital, Tianjin 300300;

${ }^{4}$ Department of Psychiatry, Tianjin Anding Hospital, Tianjin Mental Health Center, Tianjin 300222;

${ }^{5}$ Department of Radiology, Tianjin Medical University General Hospital, Tianjin 300052, P.R. China

Received August 16, 2016; Accepted October 5, 2016

DOI: $10.3892 /$ br.2016.796

\begin{abstract}
Many previous studies have reported that regional cerebral blood flow (rCBF) aberrations may be one of the pathological characteristics of depression and $\mathrm{rCBF}$ has demonstrated a certain degree of asymmetry. However, studies investigating the cerebral blood perfusion asymmetry changes of drug-naïve patients experiencing their first episode of major depression using pseudo-continuous arterial spin labeling (pCASL) are rare. Ten drug-naïve patients experiencing their first major depression episode and 15 healthy volunteers were enrolled in the current study. A novel pCASL method was applied to whole brain MRI scans of all of the samples. The Statistics Parameter Mapping and Relative Expression Software Tool software packages were used for the pre-processing and statistical analysis of the two sets of images, and the differences in the cerebral blood perfusion at the whole brain level were compared between the two groups. Compared with the healthy control group, the cerebral perfusion of the depression patients showed an asymmetric pattern. Decreased cerebral
\end{abstract}

Correspondence to: Professor Chuanjun Zhuo, Department of Psychiatry, Tianjin Anding Hospital, Tianjin Mental Health Center, 13 Liulin Road, Tianjin 300222, P.R. China

E-mail: chuanjunzhuotjmh@163.com

Professor Shengzhang Ji, Department of Radiology, Tianjin 4th Centre Hospital, 23 Zhongshan Road, Tianjin 300143, P.R. China

E-mail: zhongbaoliangwang@yeah.net

*Contributed equally

Key words: major depression, first-episode, drug naïve, magnetic resonance imaging, functional magnetic resonance imaging, pseudo continuous arterial spin labeling, pseudo-continuous arterial spin labeling, regional cerebral flow, regional cerebral blood flow, asymmetry blood perfusion regions were primarily located in the left hemisphere, specifically in the left temporal lobe, frontal lobe and cingulate cortex $[\mathrm{P}<0.05$ and cluster size $\geq 30$ with false discovery rate (FDR) correction]. Simultaneously, increased perfusion regions were predominantly located in the right hemisphere, specifically in the right cerebellum, thalamus, frontal lobe and anterior cingulate cortex $(\mathrm{P}<0.05$ and cluster size $\geq 30$, with FDR correction). Thus, pCASL may characterize the alterations in cerebral blood perfusion of patients with depression.

\section{Introduction}

Depression, primarily characterized by a persistent depressed mood, seriously threatens human health, with a suicide rate of $15-20 \%$ and a cure rate of only $\sim 30 \%$. To improve the cure rate of depression, current research has focused on investigation of the brain mechanisms of depression patients and targets for treatment (1). Many previous studies using positron emission tomography (PET) and single-photon emission computed tomography (SPECT) techniques have reported that regional cerebral blood flow (rCBF) aberrations may be one of the pathological characteristics of depression. Drevets (2) suggested that the brain $\mathrm{rCBF}$ in patients with depression showed a general tendency to decrease and that the brain regions with abnormal rCBF in depression patients predominantly included the frontal lobe, temporal lobe, the cingulate cortex, amygdala, caudate nucleus, and paralimbic system.

The application of SPECT and PET to clinical studies relatively accurately identifies the location of the affected brain areas (2). However, these techniques have certain risks, such as allergic response to the injected radioactive tracer. Such risk factors limit the use of SPECT and PET imaging in depression patients in the research and clinical settings. Arterial spin labeling (ASL) involves labeling of the hydrogen atoms in the blood, which are then used as an endogenous contrast agent; thus, no tracer injection is required, and the cerebral perfusion of the subject is monitored in a non-invasive state with high spatial resolution, good accuracy and repeatability. Based on 
these advantages, ASL technology has become the mainstream method in recent years for investigating the characteristics of rCBF in depression patients $(3,4)$.

The recently developed pseudo-continuous ASL (pCASL) technique more effectively balances the signal-to-noise ratio (SNR) than SPECT or PET techniques and has an improved labeling efficiency that is characteristic of the developing trend of the ASL technique (5). Certain studies have used pCASL to investigate the $\mathrm{rCBF}$ of treated patients with severe depression and found that cerebral blood perfusion alterations are predominantly located in the anterior cingulate cortex, right medial prefrontal cortex, temporal cortex, hippocampus, thalamus and cerebellum $(6,7)$. However, the results of the two above-mentioned studies may have been influenced by antidepressants (8). Selecting untreated patients with first-episode depression and using pCASL technology to detect the changes in the $\mathrm{rCBF}$ in depression patients would avoid the impact of confounding factors, such as antidepressants on $\mathrm{rCBF}$. Thus, the specific aberrant $\mathrm{rCBF}$ pattern in patients with depression may be accurately characterized, which would provide an objective basis for the early qualitative diagnosis of depression. However, to the best of our knowledge, no study on the rCBF of drug-naive patients with first-episode depression using pCASL technology has previously been reported.

Therefore, the pCASL technique using a 3T MR system was performed in the current study to investigate blood perfusion at the whole brain level in drug-naïve patients with first-episode major depression, with the aim of improving the understanding of the characteristics of $\mathrm{rCBF}$ in patients with depression.

\section{Subjects and methods}

Study subjects. The inclusion criteria were patients who met the DSM-IV diagnostic criteria for major depressive disorder at the first onset and were diagnosed by two senior psychiatrists in a structured clinical interview based on the DSM-IV (SCI-D) (9). The Hamilton Rating Scale for Depression (17-item version) (10) was used to assess disease severity in patients who were not taking any antidepressants or mood stabilizers. A total of 10 patients were enrolled from January to December 2015 in the current study. There were seven males and three females, with an age range of 18-60 years, a mean age of 38.70 \pm 9.41 years, and a disease duration of 2-12 months. A total of 15 healthy volunteers (control group) were recruited from the hospital staff of Tianjin Anning Hospital (Tianjin, China), and included six males and nine females with an age range of 20-59 years and a mean age of 38.42 \pm 9.12 years. Two senior psychiatrists ruled out a diagnosis of a potential mental disorder, and the volunteers were only enrolled if they did not have a positive family history of mental disorder. There were no significant differences between the two groups with regard to age or the level of education.

The common exclusion criteria were patients with a history of unconsciousness for $\geq 5 \mathrm{~min}$, a neurological disease diagnosis, severe mental illness, drug abuse, serious physical illness, or endocrine disease. Furthermore, patients who were pregnant, lactating, had participated in any other research study, had received treatment within one month, or that had any magnetic resonance imagine (MRI) contraindications were excluded.

The Ethics Committee of Tianjin 4th Central Hospital (Tianjin, China) approved the current study. Prior to experimentation, all subjects were fully informed of the experimental purposes, methods and risks of possible discomfort, and all patients provided written informed consent.

Image capture. A Philips Achieva 3.0 T TX whole body MRI system (Philips Medical Systems Nederland B.V., The Netherlands) was used to perform the scans. An 8-channel parallel coil was used to collect the signals. Subjects were placed in a supine position and wore non-magnetic earplugs to reduce the impact of noise during the experiment, and a supplied foam pad was fixed around the subject's head to reduce head movement. The scanning scope covered the whole brain, and a connecting line between the front and back of the brain served as a baseline during the scanning.

The MRI protocol order for the subjects was as follows: T1 weighted images (WI), T2WI and pCASL images. Conventional T1WI and T2WI were captured to exclude organic diseases, such as craniocerebral disorders and brain malformations. The pCASL scan included a single-shot echo-planar sequence (EPI), and the parameters were as follows: Echo time/repetition time $=4,000 \mathrm{msec} / 14 \mathrm{msec}$; matrix $=80 x 80$; layer thickness $=7 \mathrm{~mm}$; number of layers $=17$; interlayer spacing $=1 \mathrm{~mm}$; voxel size $=3 \times 3 \times 7 \mathrm{~mm}$; label spacing $=20 \mathrm{~mm}$; duration of labeling $=1,650 \mathrm{msec}$; label delay $=1,525 \mathrm{msec}$; and field of view $=240 \times 240 \mathrm{~mm}$.

Functional MRI (fMRI) data preprocessing. For the current study, common data processing software for brain functions were used, including Statistics Parameter Mapping (SPM8; Wellcome Department of Imaging Neuroscience, London, UK) and the Relative Expression Software Tool to conduct the data preprocessing. The specific process consisted of the following: i) Data format conversion. The ASL images from the scans were processed with the built-in software for digital subtraction to obtain the perfusion map, and the format was then converted to a dual file format for SPM processing. This step was performed using MRICroN software 1.40 (http://www.cabiatl. com/mricro/mricro/mricro.html). ii) Spatial normalization. The individual perfusion image was input into a standard PET template provided by SPM8. iii) Removal of non-brain tissues. The normalized image was computed with a standard mask to remove the non-brain tissue. This process was achieved using the ImCalc function of SPM8. iv) Gaussian smoothing. A Gaussian kernel of $4 \times 4 \times 4 \mathrm{~mm}^{3}$ full width at half maximum (FWHM) was used to perform the spatial smoothing of the images, reduce the remaining inter-individual differences after the normalization, and improve the SNR. v) The mean of the whole brain perfusion was extracted to obtain the normalized image for individuals. The inter-individual differences in brain perfusion were removed using the individual whole brain perfusion information/average individual whole brain perfusion information. This was achieved using the REST and the ImCalc functions of SPM8.

Statistical analysis. SPM8 was used to conduct the statistical analysis of the data of brain perfusion in the two groups 
Table I. Social and demographic data for all participants.

\begin{tabular}{lccrr}
\hline Variable & Patient group $(\mathrm{n}=10)$ & Control group $(\mathrm{n}=15)$ & t-value & P-value \\
\hline Age (years) & $38.7 \pm 9.4$ & $38.4 \pm 9.1$ & 0.350 & 0.557 \\
Education level (years) & $12.7 \pm 2.1$ & $12.6 \pm 2.2$ & 0.041 & 0.840 \\
HAMD-17 score & $35.3 \pm 6.9$ & $4.6 \pm 2.3$ & 17.427 & 0.000 \\
\hline
\end{tabular}

Table II. Comparison of brain regions with decreased cerebral perfusion between patients with depression and the healthy control subjects.

\begin{tabular}{lcccc}
\hline Brain region & Cluster size & MNI coordinates (x, y, z) & $\mathrm{t}$-value & Brodmann area location \\
\hline Left temporal lobe & 50 & $-24,16,-46$ & -6.7440 & 38 \\
Right parietal lobe & 31 & $66,-4,26$ & -6.8639 & 6 \\
Left frontal lobe and cingulate cortex & 32 & $-20,-40,32$ & -7.6494 & - \\
Left paracentral lobule and precentral gyrus & 119 & $-14,-24,74$ & -8.2019 & 6,4 \\
\hline
\end{tabular}

MNI, Montreal Neurological Institute.

and to present the results. An independent one-sample t-test was conducted for the healthy control and patient groups, with age and gender as the statistical covariates to produce the difference map of cerebral blood perfusion between the two groups. The results were superimposed onto a single T1 template using the $x j$ View plug-in. The brain regions demonstrating statistical significance $[\mathrm{P}<0.05$, with false discovery rate (FDR) correction and cluster size $>30$ voxels] were noted. Montreal Neurological Institute coordinate positioning (x, y, z; http://www.mcgill.ca/neuro/) and Brodmann area (http://www. talairach.org) identification were performed, and the intensity of the $\mathrm{rCBF}$ was recorded (presented by the statistical value ' $t$ ' in the t-test). The rCBF images of the healthy controls were first displayed layer by layer, followed by the cerebral perfusion images of the two groups displayed in layers.

SPSS 19.0 statistical analysis software (SPSS, Inc., Chicago, IL, USA) was used for the input, sorting and statistical analysis of the basic data for the two groups. The statistical analysis was performed using two independent sample t-tests, and $\mathrm{P}<0.05$ was considered to indicate a statistically significant difference. The data are presented as the mean \pm standard deviation.

\section{Results}

Social and demographic data of the participants. Ten patient participants were enrolled in the current study, including seven males and three females, with an age range of 18-60 years and a disease duration of 2-12 months. Simultaneously, 15 healthy volunteers were enrolled in the study, including six males and nine females, with an age range of 20-59 years. The detailed clinical information is presented in Table I.

Comparison of the brain regions with altered cerebral perfusion between patients with depression and the healthy control group. In the current study, increased and decreased CBF brain regions were observed in the untreated depression patients when compared with the healthy controls. The significantly decreased cerebral perfusion brain regions were located in the left temporal lobe, left frontal lobe, left cingulate cortex and right parietal lobe $(\mathrm{P}<0.05$ and cluster size $\geq 30$, with FDR correction; Table II and Fig. 1). Additionally, the significantly increased cerebral perfusion regions were located in the right cerebellum, right thalamus, right frontal lobe and right anterior cingulate cortex $(\mathrm{P}<0.05$ and cluster size $\geq 30$, with FDR correction; Table III and Fig. 2). Overall, the decreased cerebral perfusion regions were primarily located in the left hemisphere, with the exception of the right parietal lobe, and the increased cerebral perfusion regions were predominantly located in the right hemisphere.

Comparison of the brain regions with altered gray matter volume between patients with depression and the healthy control group. In the current study, the gray matter volume alterations in the patients with first-episode untreated depression were investigated. Although the gray matter volume was found to be decreased in the bilateral frontal lobe, bilateral occipital lobe, left thalamus and left cerebellum, the significance of these findings disappeared following FDR correction.

\section{Discussion}

The best of our knowledge, the current investigation is the first to use advanced pCASL technology to characterize specific alterations in the rCBF in first-episode drug-naïve depression patients. Compared with previous studies, the findings strictly controlled the influence of therapeutic agents and more objectively characterized the specific alterations of $\mathrm{rCBF}$ in the patients with depression. The most notable finding of our current study is that the alterations of rCBF in drug-naïve patients with first-episode depression tended to display an asymmetric pattern. Almost all of the decreased cerebral blood perfusion brain regions were located in the left hemisphere, 
Table III. Comparison of the brain regions with increased cerebral perfusion between patients with depression and the healthy control subjects.

\begin{tabular}{lcccc}
\hline Brain region & Cluster size & MNI coordinates (x, y, z) & t-value & Brodmann area location \\
\hline Right cerebellum (posterior lobe) & 50 & $62,-68,-48$ & 4.6746 & - \\
Right cerebellum (tonsil) & 36 & $2,-54,-46$ & 5.8710 & - \\
Right thalamus & 57 & $4,-10,2$ & 7.9571 & - \\
Right frontal lobe, anterior cingulate cortex & 38 & $20,46,6$ & 6.8155 & 32 \\
Right anterior cingulate cortex & 30 & $18,36,24$ & 7.0385 & 32 \\
\hline
\end{tabular}

MNI, Montreal Neurological Institute.

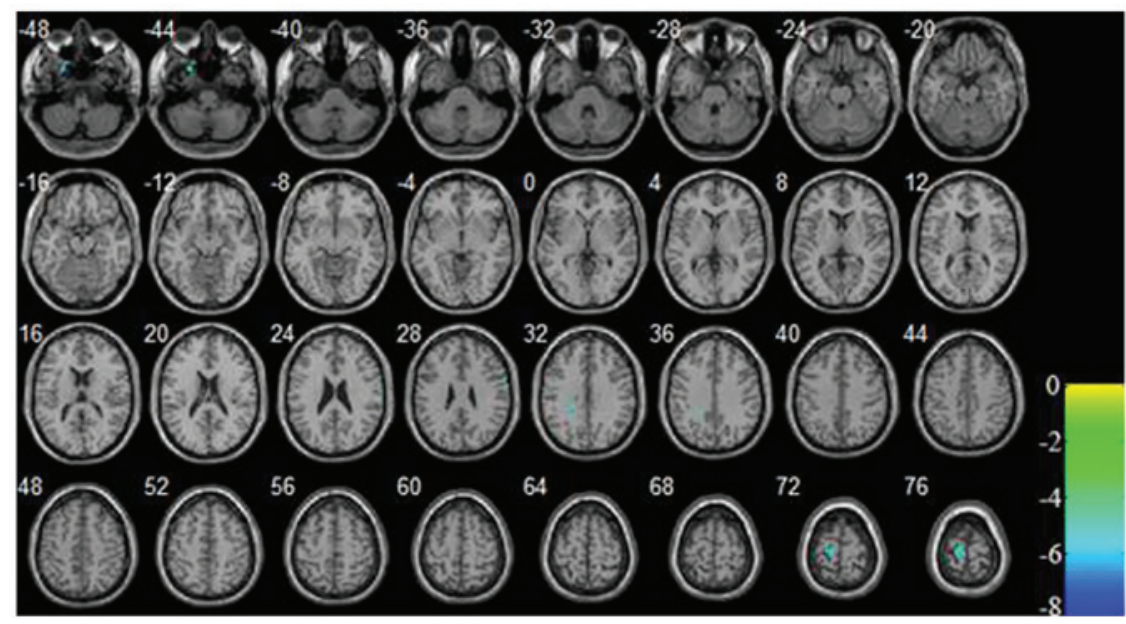

Figure 1. Images displayed in layers for the brain regions with reduced brain perfusion in patients with depression vs. the healthy control group. $\mathrm{P}<0.05$ and cluster size $\geq 30$ (with false discovery rate correction).

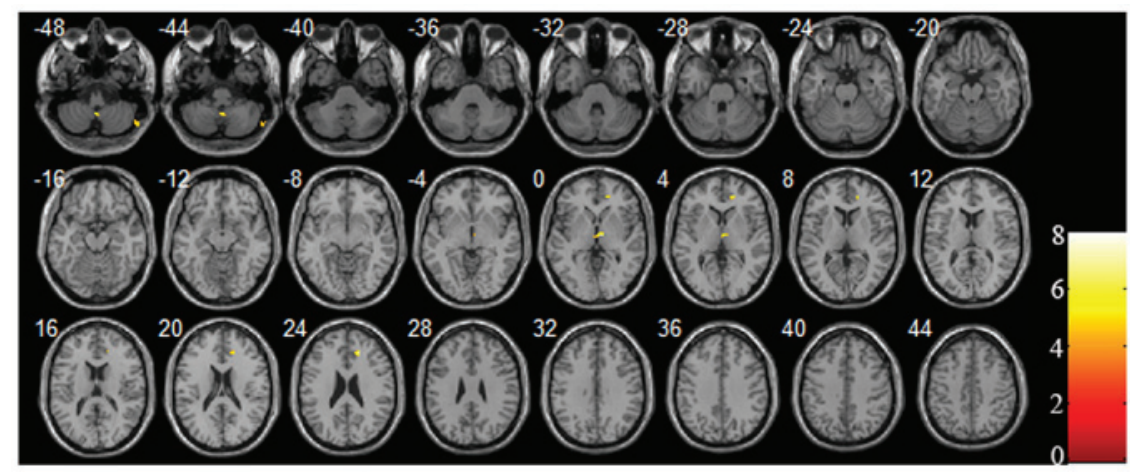

Figure 2. Images displayed in layers for the brain regions with increased perfusion in patients with depression vs. the healthy control group. $\mathrm{P}<0.05$ and cluster size $\geq 30$ (with false discovery rate correction).

specifically the left prefrontal cortex, left temporal lobe, and left cingulate cortex, with the exception of the right parietal lobe. By contrast, all the increased cerebral perfusion brain regions were located in the right hemisphere, specifically the right cerebellum, right thalamus, right frontal lobe and right anterior cingulate cortex. According to previous studies, the prefrontal cortex, temporal lobe, cingulate cortex, parietal lobe, thalamus and cerebellum had been confirmed to be key in emotional perception, memory, integration, regulation and expression processing (6,7,11-13). The $\mathrm{rCBF}$ alterations in these brain regions cause emotional disturbances and are proposed to be the neural substrate of depression.

In the current study, the asymmetric pattern of the $\mathrm{rCBF}$ alterations in drug-naïve first-episode depression patients were supported by other studies using different techniques. For example, a study using 320-Slice Computed Tomography found that the $\mathrm{rCBF}$ of the left hemisphere is lower than that in the right hemisphere in depression patients (14), although the present study did not find that $\mathrm{rCBF}$ increased in the right hemisphere in depression patients compared with the healthy 
control subjects. Additionally, numerous electrophysiology studies confirmed that brain electrical activity also exhibits an asymmetric pattern. For example, a decreased pattern was observed in left cerebral electrical activity, and decreased left hemisphere activity may influence the ability of the subject to modulate emotion, which is associated with depressive episodes (15-18). Furthermore, certain studies found that hypo-neural activity is predominantly exhibited in the left brain hemisphere and that hyper-neural activity is predominantly exhibited in the right brain hemisphere in depression patients. For example, a study using an event related potential technique found that depression patients exhibited lower activation in the central and left brain regions (19). Another study found that electrophysiologic power asymmetry was present in the depression patients, and that the right brain hemisphere of depression patients showed higher $\theta$ power when compared with the healthy control subjects (20). Furthermore, a meta-analysis of depression fMRI studies indicated that hypo-activity or hypo-connectivity of brain regions was primarily localized to the left hemisphere (21). Notably, a previous study found that neural electrical activity alterations were associated with rCBF alterations (22). Together, these previous studies and the current findings suggest that a functional asymmetric alteration pattern is present in depression patients. Although this hypothesis has no substantial basis and may seem unlikely, it provides a point of reference for additional studies of brain function in depression patients.

Cerebral blood perfusion was found to be significantly decreased in the left brain hemisphere, which is consistent with the findings of previous studies $(23,24)$. However, in the present study, cerebral blood hyper-perfusion tended to be located in the right brain hemisphere, which is inconsistent with certain previous studies $(7,25)$. Our current findings and the findings of previous studies indicate a sophisticated phenomenon; however, this is not surprising, as the results of the different studies may have been influenced by various confounding factors, such as differences in clinical characteristics of the participants, effects of antidepressants on the participants, and the technical characteristics of image acquisition and analysis. Given all of these potential confounding factors, to the best of our knowledge, there are currently no consistent findings. Accurately addressing the question of functional brain asymmetry changes in patients with depression requires a large sample and long-term follow up studies of drug-naïve first-episode depression patients. These patients must be enrolled using uniform diagnosis criteria for uniform neuroimaging acquisition and analysis to compare rCBF measures between patients and healthy controls. In addition to large-sample long-term follow up studies, rCBF measures should be compared before and after treatment to investigate the association between the alterations of rCBF and the effects of antidepressants. Thus, improving our understanding of the mechanisms of depression and establishing an objective predictor of antidepressant effects may facilitate the development of tailored treatments and personalized medicine (26).

In conclusion, the $\mathrm{rCBF}$ perfusion alterations in drug-naïve first-episode depression patients displayed an asymmetric pattern, where decreased cerebral blood perfusion was predominantly localized to the left hemisphere and increased
rCBF was predominantly localized to the right hemisphere. The altered $\mathrm{rCBF}$ brain regions overlapped with brain centers associated with emotional processing. Thus, the pCASL technique revealed specific alteration patterns that are characteristic of rCBF perfusion in drug-naïve patients with first-episode depression and may be used as an auxiliary objective indicator for clinical diagnosis.

\section{Acknowledgements}

The present study was supported by grants from the Science and technology fund of Tianjin Health Bureau (grant no. 2013KR03 to Haiman Bian).

\section{References}

1. Saltiel PF and Silvershein DI: Major depressive disorder: Mechanism-based prescribing for personalized medicine. Neuropsychiatr Dis Treat 11: 875-888, 2015.

2. Drevets WC: Neuroimaging studies of mood disorders. Biol Psychiatry 48: 813-829, 2000.

3. Ho TC, Wu J, Shin DD, Liu TT, Tapert SF, Yang G, Connolly CG Frank GK, Max JE, Wolkowitz O, et al: Altered cerebral perfusion in executive, affective, and motor networks during adolescent depression. J Am Acad Child Adolesc Psychiatry 52: 1076-1091.e2, 2013.

4. Wang DJ, Chen Y, Fernández-Seara MA and Detre JA: Potentials and challenges for arterial spin labeling in pharmacological magnetic resonance imaging. J Pharmacol Exp Ther 337: 359-366, 2011.

5. Hartkamp NS, Petersen ET, De Vis JB, Bokkers RP and Hendrikse J: Mapping of cerebral perfusion territories using territorial arterial spin labeling: Techniques and clinical application. NMR Biomed 26: 901-912, 2013.

6. Järnum H, Eskildsen SF, Steffensen EG, Lundbye-Christensen S Simonsen CW, Thomsen IS, Fründ ET, Théberge J and Larsson EM: Longitudinal MRI study of cortical thickness, perfusion, and metabolite levels in major depressive disorder. Acta Psychiatr Scand 124: 435-446, 2011.

7. Ota M, Noda T, Sato N, Hattori K, Teraishi T, Hori H, Nagashima A, Shimoji K, Higuchi T and Kunugi H: Characteristic distributions of regional cerebral blood flow changes in major depressive disorder patients: A pseudo-continuous arterial spin labeling (pCASL) study. J Affect Disord 165: 59-63, 2014.

8. Nobler MS, Olvet KR and Sackeim HA: Effects of medications on cerebral blood flow in late-life depression. Curr Psychiatry Rep 4: 51-58, 2002.

9. First MB, Spitzer RL, Gibbon M and Williams JB: Structured Clinical Interview for DSM-IV Axis I Disorders (SCID). Biometrics Research, New York State Psychiatric Institute, New York, NY, 1996.

10. Hamilton M: A rating scale for depression. J Neurol Neurosurg Psychiatry 23: 56-62, 1960.

11. Alexopoulos GS, Kiosses DN, Heo M, Murphy CF, Shanmugham B and Gunning-Dixon F: Executive dysfunction and the course of geriatric depression. Biol Psychiatry 58: 204-210, 2005.

12. Davies J, Lloyd KR, Jones IK, Barnes A and Pilowsky LS: Changes in regional cerebral blood flow with venlafaxine in the treatment of major depression. Am J Psychiatry 160: 374-376, 2003.

13. Galynker II, Cai J, Ongseng F, Finestone H, Dutta E and Serseni D: Hypofrontality and negative symptoms in major depressive disorder. J Nucl Med 39: 608-612, 1998.

14. Wang Y, Zhang H, Tang S, Liu X, O'Neil A, Turner A, Chai F, Chen $\mathrm{F}$ and Berk M: Assessing regional cerebral blood flow in depression using 320-slice computed tomography. PLoS One 9: e107735, 2014.

15. Blackhart GC, Minnix JA and Kline JP: Can EEG asymmetry patterns predict future development of anxiety and depression? A preliminary study. Biol Psychol 72: 46-50, 2006.

16. Bruder GE, Quitkin FM, Stewart JW, Martin C, Voglmaier MM and Harrison WM: Cerebral laterality and depression: Differences in perceptual asymmetry among diagnostic subtypes. J Abnorm Psychol 98: 177-186, 1989. 
17. Hannesdóttir DK, Doxie J,Bell MA, Ollendick TH and Wolfe CD: A longitudinal study of emotion regulation and anxiety in middle childhood: Associations with frontal EEG asymmetry in early childhood. Dev Psychobiol 52: 197-204, 2010.

18. Kemp AH, Cooper NJ, Hermens G, Gordon E, Bryant R and Williams LM: Toward an integrated profile of emotional intelligence: Introducing a brief measure. J Integr Neurosci 4: 41-61, 2005.

19. Liu H, Yin HF, Wu DX and Xu SJ: Event-related potentials in response to emotional words in patients with major depressive disorder and healthy controls. Neuropsychobiology 70: 36-43, 2014.

20. Quraan MA, Protzner AB, Daskalakis ZJ, Giacobbe P, Tang CW, Kennedy SH, Lozano AM and McAndrews MP: EEG power asymmetry and functional connectivity as a marker of treatment effectiveness in DBS surgery for depression. Neuropsychopharmacology 39: 1270-1281, 2014.

21. Sundermann B, Olde Lütke Beverborg M and Pfleiderer B: Toward literature-based feature selection for diagnostic classification: A meta-analysis of resting-state fMRI in depression. Front Hum Neurosci 8: 692, 2014.
22. Moretti DV, Prestia A, Binetti G, Zanetti O and Frisoni GB: Increase of theta frequency is associated with reduction in regional cerebral blood flow only in subjects with mild cognitive impairment with higher upper alpha/low alpha EEG frequency power ratio. Front Behav Neurosci 7: 188, 2013

23. Lui S, Parkes LM, Huang X, Zou K, Chan RC, Yang H, Zou L, Li D, Tang H, Zhang T, et al: Depressive disorders: Focally altered cerebral perfusion measured with arterial spin-labeling MR imaging. Radiology 251: 476-484, 2009.

24. Smith DJ and Cavanagh JT: The use of single photon emission computed tomography in depressive disorders. Nucl Med Commun 26: 197-203, 2005.

25. Vasic N, Wolf ND, Grön G, Sosic-Vasic Z, Connemann BJ, Sambataro F, von Strombeck A, Lang D, Otte S, Dudek M, et al: Baseline brain perfusion and brain structure in patients with major depression: A multimodal magnetic resonance imaging study. J Psychiatry Neurosci 40: 412-421, 2015.

26. Patrick KS, Corbin TR and Murphy CE: Ethylphenidate as a selective dopaminergic agonist and methylphenidate-ethanol transesterification biomarker. J Pharm Sci 103: 3834-3842, 2014 